\title{
Cisto hepático benigno simulando metástase de carcinoma diferenciado de tireoide
}

\author{
Benign hepatic cyst mimicking thyroid carcinoma metastasis
}

Ana Paula Cançado Gonçalves', Carolina Souza Jorge ${ }^{2}$, Jader Pereira Resende', José Ribamar Silva Villela ${ }^{3}$, Maria Marta Sarquis Soares ${ }^{2,4}$, Adauto Versiani Ramos ${ }^{1}$

\begin{abstract}
RESUMO
Introdução: Pesquisa de corpo inteiro após dose terapêutica de (131I) Nal (PCI) associada à tireoglobulina (Tg) sérica, anticorpo antitireoglobulina (anti-Tg) e ultrassom (US) cervical representam os métodos de referência para detecção de carcinoma diferenciado de tireoide (CDT) residual ou metastático. Algumas causas de $\mathrm{PCl}$ falsos-positivas, como processos inflamatórios, alguns tumores não tireoidianos e até mesmo cistos renais e sebáceos, são bem conhecidas. Métodos: Neste trabalho, descreveu-se um caso de cisto hepático benigno simulando metástase de carcinoma de tireoide em PCl após dose terapêutica de (131) Nal. Resultados: Ressalta-se a importância do reconhecimento dos cistos hepáticos benignos como fator complicador do seguimento dos pacientes com câncer de tireoide. Conclusões: Para minimizar erros de diagnóstico e, consequentemente, na condução dos casos de CDT, é necessário conhecer as possíveis causas de PCl falsos-negativas e positivas. Arq Bras Endocrinol Metab. 2009;53(6):777-82
\end{abstract}

Descritores

Cisto hepático; pesquisa de corpo inteiro após (1311) Nal; carcinoma tireoidiano

\begin{abstract}
Introduction: The follow-up of differentiated thyroid carcinoma (DTC) for detecting persistent or recurrent disease is based on iodine whole body scan (WBS), the evaluation of the tumor marker thyroglobulin $(\mathrm{Tg})$, the anti-thyroglobulin antibody (anti-Tg) and neck ultrasonography (US). Well known false-positive causes of WBS include inflammatory processes, some non-thyroid tumors, kidney or even sebaceous cysts . Methods: We reported a case of falsepositive WBS, after therapeutic dose of (131) Nal. Results: We enphasize the importance of recognizing benign liver cysts mimicking DTC metastasis. Conclusions: False-positive and negative results may occur with WBS and must be recognized to avoid mismanagement. Arq Bras Endocrinol Metab. 2009;53(6):777-82
\end{abstract}

\author{
${ }^{1}$ Serviço de Endocrinologia \\ do Hospital Felício Rocho, \\ Belo Horizonte, MG, Brasil \\ ${ }^{2}$ Universidade Federal de \\ Minas Gerais (UFMG), Belo \\ Horizonte, MG, Brasil \\ ${ }^{3}$ Departamento de Medicina \\ Nuclear do Laboratório Hermes \\ Pardini, Belo Horizonte, MG, Brasil \\ ${ }^{4}$ Instituto Felício Rocho de Pesquisa \\ e Educação Continuada (Iferpec) \\ Belo Horizonte, MG, Brasil
}

Correspondência para: Ana Paula Cançado Gonçalves Av. Sete de Setembro, 1.298, apto. 702 - Centro

35500-011 - Divinópolis, MG, Brasil aninhacancado@hotmail.com

Recebido em 22/Out/2008 Aceito em 20/Mai/2009

\section{INTRODUÇÃO}

$\mathrm{O}$ câncer de tireoide, apesar de responder pela maior parte das neoplasias malignas endócrinas, representa apenas cerca de $1 \%$ de todos os cânceres humanos (1). Os carcinomas diferenciados são responsáveis por aproximadamente $90 \%$ de todos os cânceres de tireoide, sendo $90 \%$ desses representados pelo carcinoma papilar de tireoide (CPT) (1-3).

O CPT acomete pacientes de qualquer idade e sexo, embora apresente pico de incidência entre 30 e 50 anos e ocorra, principalmente, em mulheres $(60 \%$ a $80 \%$ dos casos) (3).

A disseminação do CPT ocorre por meio de linfáticos intraglandulares, evoluindo do foco inicial para tecidos moles peritireoidianos, linfonodos pericapsulares e cervicais (3). Cerca de um terço dos pacientes com CPT apresentam linfadenopatia clinicamente evidente ao diagnóstico, e $35 \%$ a $50 \%$ dos linfonodos retirados da região cervical possuem evidência histológica de envolvimento neoplásico (3). Já a invasão extranodal de tecidos moles 
adjacentes está presente em aproximadamente $15 \%$ dos casos, por ocasião da cirurgia primária. Os sítios de metástases a distância encontrados em pacientes com carcinoma diferenciado da tiroide (CDT) são: pulmão (49\%), osso (24\%), sítios múltiplos (19\%) e outros sítios únicos $(8 \%)$ (4). Já para CPT, somente $1 \%$ a $7 \%$ dos pacientes apresentam metástases a distância ao diagnóstico (3), sendo pulmão e mediastino os sítios mais comuns (5).

Além da forma clássica do CPT, são conhecidos alguns subtipos do carcinoma papilar que respondem por $20 \%$ de todos os casos: variante folicular, variante de células altas, variante de células colunares e variante esclerosante difusa. Importante ressaltar que as variantes de células altas, células colunares e esclerosante difusa constituem os subtipos mais agressivos (3). O subtipo variante esclerosante difusa foi descrito pela primeira vez em 1985 e responde por $2 \%$ a $6 \%$ de todos os CPT $(6,7)$. Essa variante do CPT ocorre mais comumente entre as mulheres, sendo 27 anos a idade média dos pacientes ao diagnóstico (6). A incidência de metástases linfonodais e pulmonares é significativamente maior na variante esclerosante difusa do que na forma clássica do CPT $(7,8)$. Metástases linfonodais são encontradas em mais de $70 \%$ dos pacientes acometidos pela variante (6). Apesar disso, o prognóstico da variante esclerosante difusa do CPT permanece controverso na literatura. Estudos defendem que esse subtipo do CPT, em razão das características acima descritas, apresenta pior prognóstico em relação à forma clássica (8). No entanto, estudos mais recentes são menos enfáticos nessa afirmação ou até sustentam que o prognóstico é similar entre a variante esclerosante difusa e a forma clássica do CPT $(6,7)$. Diante dessa questão, são necessários novos estudos envolvendo tanto maior número de pacientes quanto tempo de seguimento. O que parece nunca ter deixado de ser consenso, em relação ao carcinoma papilar variante esclerosante difusa, é a indicação de tireoidectomia total com ressecção em bloco de estruturas infiltradas combinada com dissecção regional ampla de linfonodos, seguida, invariavelmente, de terapia com radioiodo $(6,7,8)$.

A dosagem da tireoglobulina $(\mathrm{Tg})$ acompanhada da dosagem do anticorpo antitireoglobulina (anti-Tg), do ultrassom (US) cervical e da pesquisa de corpo inteiro após dose terapêutica de ( $\left.{ }^{131} \mathrm{I}\right) \mathrm{NaI}$ (PCI) são, hoje, os métodos de referência para detectar CDT residuais e/ ou metastáticos (1-3,9-13).

Os cistos hepáticos simples têm sido cada vez mais diagnosticados, uma vez que métodos de imagem, como o US, são frequentemente utilizados na atual prática clínica. Esses cistos são encontrados em $20 \%$ dos pacientes submetidos à laparotomia por outras causas. A maioria dos pacientes é assintomática e possui provas de função hepática normais (14). Ao US, os cistos hepáticos simples aparecem como estruturas de paredes finas com conteúdo anecoico, apresentando reforço acústico posterior (14). Septos finos completos ou incompletos podem ser identificados no interior desses cistos. Menos comumente, os cistos hepáticos simples podem apresentar hemorragia ou infecção e, nessas situações, o US pode mostrar conteúdo interno de espessura variável bem como septações mais espessas (14).

Relatou-se o caso de paciente portadora de cisto hepático benigno que, em PCI após dose terapêutica de ( $\left.{ }^{131} \mathrm{I}\right) \mathrm{NaI}$, mostrou captação do radiofármaco. Essa entidade precisa ser amplamente reconhecida por causa das sérias consequências de ser erroneamente diagnosticada como metástase hepática de CDT. O objetivo desse trabalho foi ressaltar que a PCI após a dose terapêutica de $\left({ }^{131} \mathrm{I}\right) \mathrm{NaI}$, apesar de ser um importante meio de acompanhamento de pacientes com CDT no pós-operatório, deve ser interpretada com cautela, já que possui causas relevantes, tanto de resultados falsosnegativos quanto positivos (10-12).

\section{RELATO DE CASO}

Paciente do sexo feminino, 66 anos de idade, procurou endocrinologista em outubro de 1999, em decorrência de dores na região cervical anterior. Ao palpar a tireoide, foi notado nódulo duro e móvel. US de tireoide confirmou nódulo hipoecogênico de contornos imprecisos, medindo $1,01 \times 0,57 \times 0,51 \mathrm{~cm}$, no istmo. A paciente foi submetida à tireoidectomia total em abril de 2000, após punção sugestiva de neoplasia folicular. $\mathrm{O}$ anatomopatológico da peça cirúrgica definiu a lesão como carcinoma papilar variante esclerosante difusa. A primeira dosagem de $\mathrm{Tg}$, no pós-operatório, foi de $50,2 \mathrm{ng} / \mathrm{mL}$ (quimioluminescência: $\mathrm{VR}=1,15$ a $35 \mathrm{ng} / \mathrm{mL}$ ) com anti- $\mathrm{Tg}$ negativo (quimioluminescência: $\mathrm{VR}<40 \mathrm{IU} / \mathrm{mL}$ ) e TSH de $46 \mu \mathrm{UI} / \mathrm{mL}$ (quimioluminescência: $\mathrm{VR}=0,34$ a $5,6 \mu \mathrm{UI} / \mathrm{mL}$ ), sendo a paciente submetida, então, ao tratamento ablativo com $100 \mathrm{mCi}(3700 \mathrm{MBq})$ de ( $\left.{ }^{131} \mathrm{I}\right)$ NaI PCI após dose terapêutica; evidenciou-se captação apenas em região cervical (Tabela 1 ). Seis meses após o tratamento, o US cervical demonstrou pequenas massas em topografia de tireoide, e a punção dessas massas sugeriu, novamente, neoplasia folicular. Foi reoperada em 


\begin{tabular}{|c|c|c|c|c|c|c|}
\hline $\begin{array}{l}\text { Data exames } \\
\text { (mês/ano) }\end{array}$ & $\mathrm{TG}(\mathbf{n g} / \mathrm{mL})$ & Anti-TG & TSH ( $\mu \mathrm{UI} / \mathrm{mL})$ & $\begin{array}{l}\text { Dose }\left({ }^{131} \mathrm{I}\right) \\
\text { Nal (mci) }\end{array}$ & PCI (captação)* & Observações \\
\hline Abril/2000 & 50,2 & Negativo & 46 & 100 & Região cervical & Pós-operatório imediato \\
\hline Janeiro/2004 & 27,7 & Negativo & 31 & 200 & $\begin{array}{l}\text { Regiões cervical anterior, } \\
\text { mediastinal, pélvica à esquerda e } \\
\text { hepática }\end{array}$ & $\begin{array}{l}\text { US* cervical: nódulos cervicais } \\
\text { US abdômen: lesão hepática }\end{array}$ \\
\hline Janeiro/2005 & 28 & Negativo & 48 & 300 & $\begin{array}{l}\text { Região cervical, mediastinal e } \\
\text { hepática }\end{array}$ & $\begin{array}{l}\text { Cintilografia óssea: lesão } \\
\text { hipercaptante no esterno } \\
\text { RNM }^{\star \star \star} \text { abdômen: cisto hepático }\end{array}$ \\
\hline Janeiro/2006 & 5,7 & Negativo & 37 & $\begin{array}{c}300 \\
\text { (associado a lítio) }\end{array}$ & $\begin{array}{l}\text { Apenas em região de topografia } \\
\text { hepática superior esquerda }\end{array}$ & $\begin{array}{l}\text { Cintilografia óssea: negativa } \\
\text { US cervical: sem alterações }\end{array}$ \\
\hline Fevereiro/2008 & 16 & Negativo & 34 & & & $\begin{array}{l}\text { Biópsia de cisto hepático (Tg e } \\
\text { citologia negativas) }\end{array}$ \\
\hline
\end{tabular}

* $\mathrm{PCl}$ após dose terapêutica de (13i!) Nal. ** Ultrassom. * ${ }^{\star \star \star}$ Ressonância nuclear magnética.

dezembro de 2000, e o anatomopatológico demonstrou apenas processo inflamatório inespecífico. A partir daí, os níveis séricos de $\mathrm{Tg}$ em supressão com levotiroxina oscilaram entre 1,6 e $2,6 \mathrm{ng} / \mathrm{mL}$, sempre com anti-Tg negativo. No início de 2004, a dosagem da Tg, com TSH de $31 \mu \mathrm{UI} / \mathrm{mL}$, foi $27,7 \mathrm{ng} / \mathrm{mL}$, o que desencadeou extensa propedêutica para rastrear prováveis metástases. US cervical visualizou imagens localizadas nas regiões paratraqueal direita e medial à carótida comum. US abdominal demonstrou imagem hipoecogênica de $4,1 \mathrm{~cm}$ no lobo esquerdo do fígado e tomografia (TC) de tórax não identificou alterações. Recebeu $200 \mathrm{mCi}$ (7400 MBq) de ( ${ }^{131}$ I) NaI e PCI após dose terapêutica; dessa vez, apresentou captação em regiões cervical anterior, mediastinal, hemipélvica esquerda e hepática. Reiniciou-se supressão com levotiroxina, com obtenção dos níveis de $\operatorname{Tg}$ de $0,7 \mathrm{ng} / \mathrm{mL}$. No início de 2005 , já sem supressão, houve recorrência do aumento da $\mathrm{Tg}$ para 28 ng/mL (TSH: $48 \mu \mathrm{UI} / \mathrm{mL}$ ). À ressonância nuclear magnética (RNM), foi visualizada formação cística hepática de paredes finas medindo $5,2 \times 4,2 \times 4,5 \mathrm{~cm}$ e, à cintilografia óssea, lesão hipercaptante no terço médio do esterno, sugerindo outro foco de metástase. Paciente recebeu, então, a terceira dose de $\left({ }^{131} \mathrm{I}\right) \mathrm{NaI}$, dessa vez $300 \mathrm{mCi}$ (11100 MBq). PCI após dose terapêutica, nessa ocasião, elucidou captação em regiões cervical, mediastinal e intensa captação em parênquima hepático (Figura 1). Reiniciada supressão com levotiroxina, houve queda da Tg para $0,5 \mathrm{ng} / \mathrm{mL}$. Em 2006, Tg com $\mathrm{TSH}$ de $37 \mu \mathrm{UI} / \mathrm{mL}$ voltou a subir para $5,7 \mathrm{ng} / \mathrm{mL}$. US cervical e US abdominal não evidenciaram alterações em relação aos anteriores. A cintilografia foi repetida e não mais evidenciou lesão hipercaptante no terço médio do esterno.

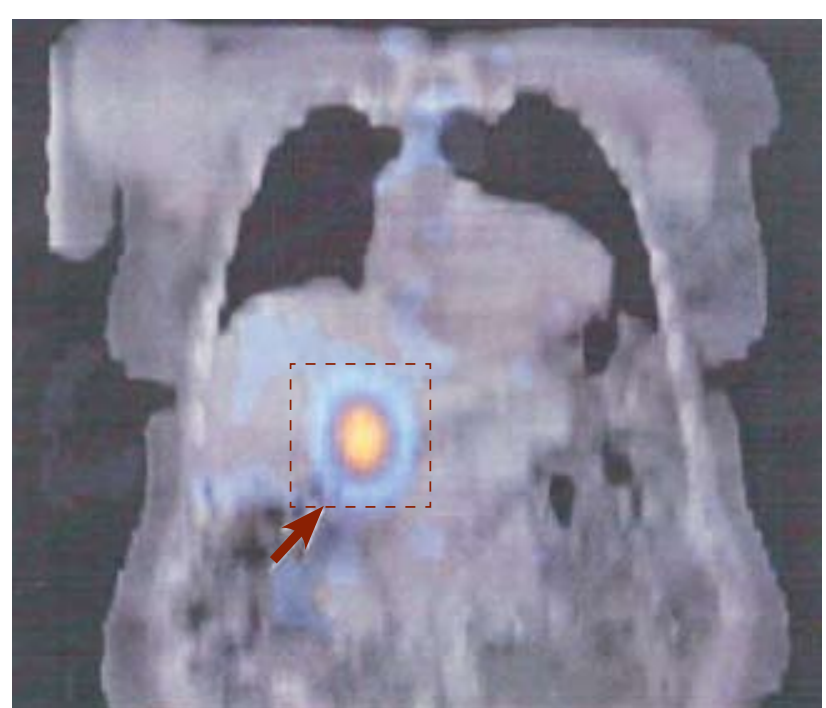

Figura 1. Imagem de fusão anatômico-funcional (SPECT-CT) com (131) Nal em lesão de cisto hepático simples evidenciando acúmulo do radiotraçador.

Após receber a quarta dose de $\left({ }^{131} \mathrm{I}\right) \mathrm{NaI}, 300 \mathrm{mCi}$ (11100 MBq) associada a lítio, na dose de $300 \mathrm{mg}$, três dias antes da PCI e cinco dias depois, foi mantida captação apenas em região hepática superior esquerda. Três meses depois, nova dosagem da $\mathrm{Tg}$, em supressão, foi de $0,75 \mathrm{ng} / \mathrm{mL}$. Em fevereiro de 2008, diante de nova $\mathrm{Tg}$ de $16 \mathrm{ng} / \mathrm{mL}$ em vigência de TSH de 34 $\mu \mathrm{UI} / \mathrm{mL}$ associada à ausência de outros sítios suspeitos de metástases, exceto a lesão hepática persistentemente captante de ( $\left.{ }^{131} \mathrm{I}\right) \mathrm{NaI}$, optou-se por punção dessa lesão, guiada por US, avaliação citológica e dosagem de Tg no material aspirado. O US para punção da lesão (Figura 2) mostrou, novamente, presença de formação cística de paredes levemente espessas e irregulares, conteúdo anecoico, medindo cerca de 5,6 x 4,2 cm, no lobo esquerdo do fígado. Foram aspirados $38 \mathrm{~mL}$ de líquido amarronzado turvo, no qual a dosagem da $\mathrm{Tg}$ foi 


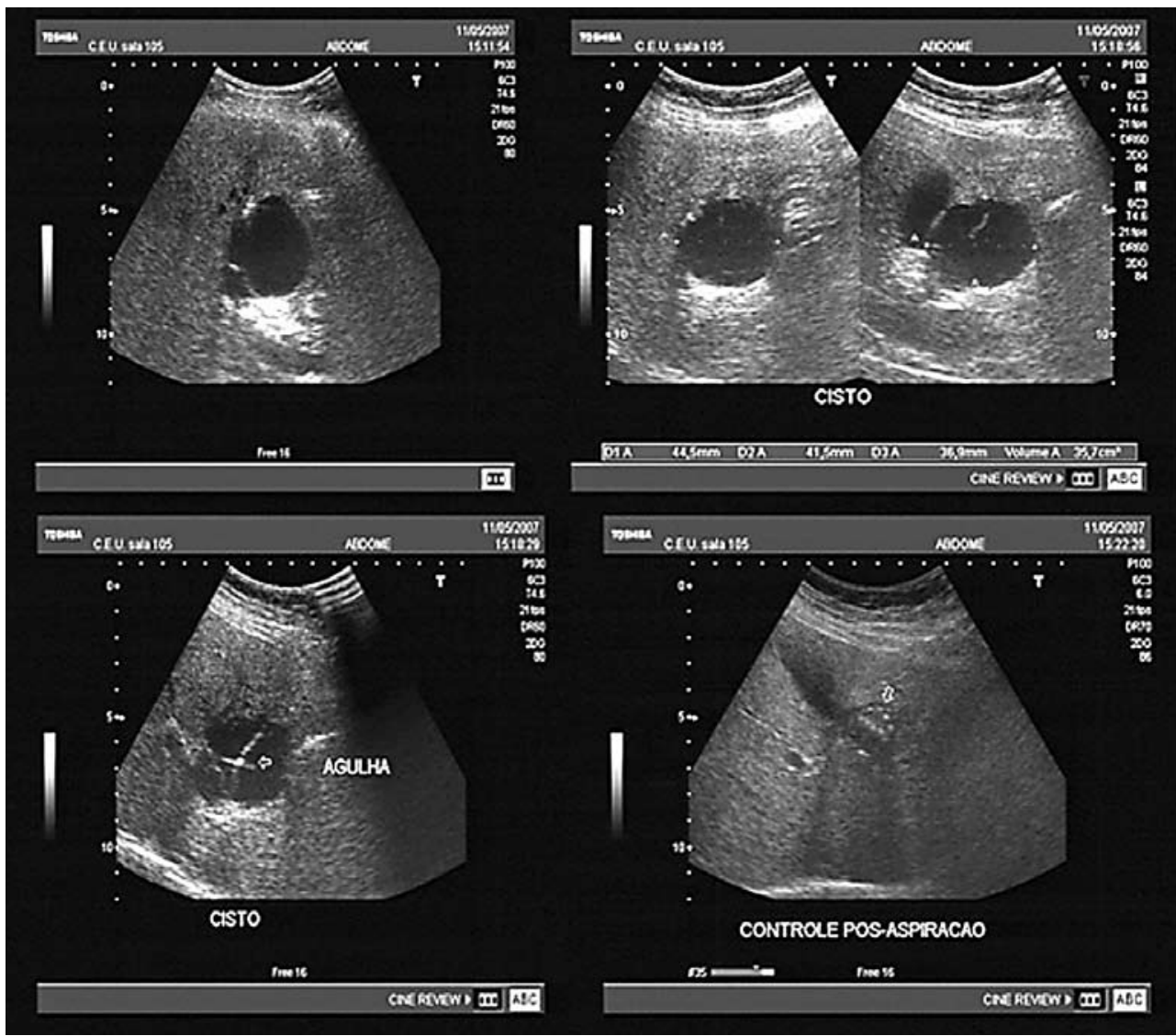

Figura 2. US abdominal: formação cística de contornos bem definidos, com finas septações, medindo cerca de 5,6 x 4,2 cm, no lobo esquerdo do fígado. Realizada punção ecoguiada do cisto hepático com aspiração subtotal do conteúdo líquido.

negativa, e o exame citológico não demonstrou atipias, não sendo observada neoplasia no material enviado. Em abril de 2008, nova cintilografia óssea foi negativa para metástases, e o valor de Tg, com TSH suprimido, foi de $0,38 \mathrm{ng} / \mathrm{mL}$. Em julho de 2008, primeiro US abdominal após punção (Figura 3 ) demonstrou retorno da imagem cística no lobo esquerdo do fígado, na mesma topografia e com as mesmas características da lesão previamente puncionada.

\section{DISCUSSÃO}

A dosagem sérica de Tg e anticorpo anti-Tg, o US cervical e a PCI após dose terapêutica de ( $\left.{ }^{131} \mathrm{I}\right) \mathrm{NaI}$ são métodos bem estabelecidos para o seguimento de pacientes depois do tratamento cirúrgico de CDT (1-3,913). Entretanto, apesar de serem exames amplamente utilizados e de inquestionável utilidade no acompanhamento desses pacientes, vale ressaltar que podem apresentar resultados tanto falsos-negativos quanto positivos, acarretando problemas diagnósticos e condutas equivocadas (10-12) (Tabela 2$)$.

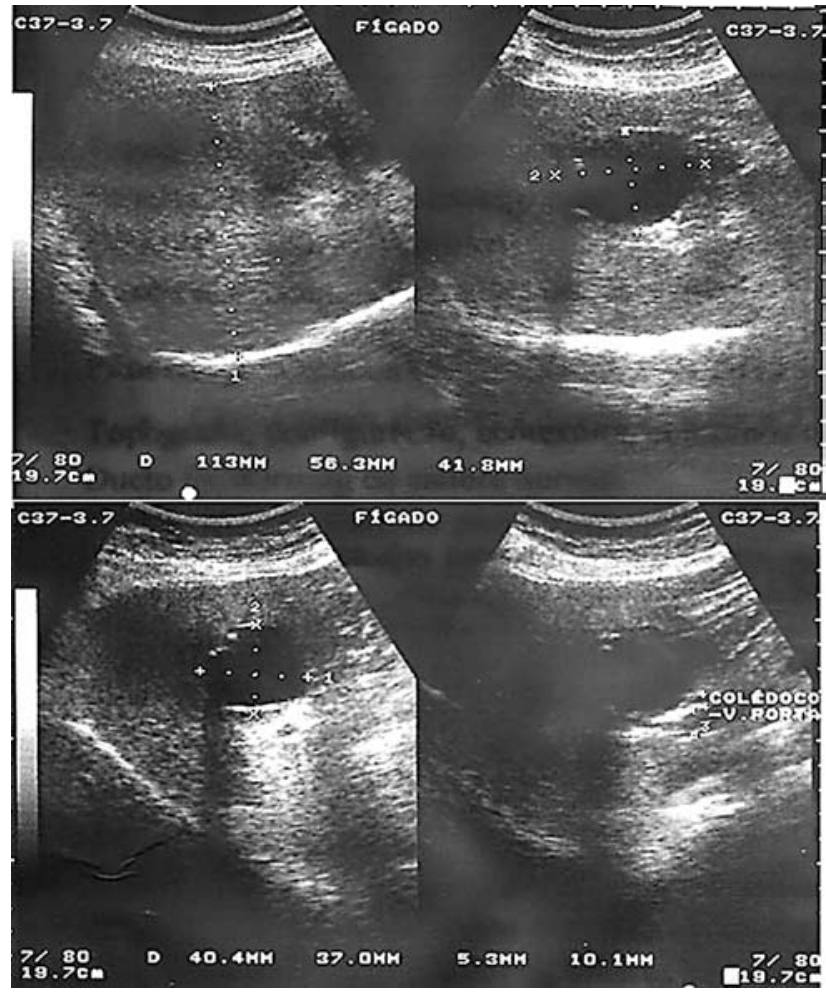

Figura 3. US abdominal para controle evolutivo cinco meses pós-punção do cisto hepático, demonstrando reenchimento desse, com as mesmas características prévias. 
Tabela 2. Condições não tireoidianas associadas com PCI falso-positiva

\begin{tabular}{ll}
\hline Contaminação & Pele, cabelo, roupas \\
\hline Secreções fisiológicas & $\begin{array}{l}\text { Glândula salivar, secreção e mucosa nasal, escarro } \\
\text { (traqueostomia), mucosa gástrica, cólon (no } \\
\text { hipotireoidismo), bexiga, mama (mulheres jovens) }\end{array}$ \\
$\begin{array}{ll}\text { Processos } \\
\text { inflamatórios }\end{array}$ & $\begin{array}{l}\text { Pulmonar, brônquico, cutâneo, dental, seios da face, } \\
\text { vesícula biliar, pericárdio }\end{array}$ \\
Outras condições & Neoplasias não tireoidianas: glândula salivar, \\
& estômago, pulmão, meningioma, Struma ovarii, \\
& teratomas \\
& Timo: normal ou hiperplásico \\
& Esôfago: dilatação, hérnia hiatal \\
& Insuficiência cardíaca, derrame pericárdico, ectasia da \\
& artéria carótida comum com estase \\
& Pele: psoríase, queimadura \\
& Cistos: renal, pleuropericárdico, salivar, mamário, \\
& hidrocele, sebáceo e hepático
\end{tabular}

A Tg é uma glicoproteína produzida somente por células tireoidianas foliculares normais ou neoplásicas (3). A dosagem de $\mathrm{Tg}$ obtida por ensaio imunorradiométrico é o marcador mais sensível e específico em detectar CDT, sobretudo após tireoidectomia total e ablação com ( $\left.{ }^{131} \mathrm{I}\right) \mathrm{NaI}$, principalmente sob estímulo do TSH ou após o uso de TSH recombinante (rhTSH) (15). Contudo, a medida de Tg com TSH estimulado é passível de não identificar tumores clinicamente significativos, em decorrência da presença de anti-Tg ou, menos comumente, da produção e da secreção deficiente ou ausente da $\mathrm{Tg}$ imunorreativa pelas células tumorais (3). Por outro lado, a Tg mensurada em supressão do TSH pode falhar em identificar pacientes com tumores residuais ou metastáticos muito pequenos (3).

Já os resultados da PCI dependem da habilidade do tecido tireoidiano em captar e reter ( $\left.{ }^{131} \mathrm{I}\right) \mathrm{NaI}$ em presença de altas concentrações séricas de TSH (16). A sensibilidade da PCI após dose terapêutica de ( $\left.{ }^{131} \mathrm{I}\right)$ $\mathrm{NaI}$ é relatada em torno de $45 \%$ a $75 \%$, dependendo do estudo (15). Resultados falsos-negativos, mesmo após dose terapêutica de ( $\left.{ }^{131} \mathrm{I}\right) \mathrm{NaI}$, podem ocorrer em razão da presença de micrometástases ou da perda da capacidade da célula neoplásica em concentrar iodo, fato decorrente da desdiferenciação das células tumorais (15). Já PCI após dose terapêutica de ( $\left.{ }^{131} \mathrm{I}\right) \mathrm{NaI}$ falsospositivas podem ser vistas em virtude da aquisição, por alguns tecidos corporais não tireoidianos, da capacidade de captar e/ou eliminar ( $\left.{ }^{131} \mathrm{I}\right) \mathrm{NaI}$ (10). Essa capacidade é descrita em alguns fluidos corporais, processos inflamatórios e infecciosos, tumores não tireoidianos e também cistos ou transudatos (10-12).
Em extensa revisão na literatura, pôde-se verificar que cisto hepático simples benigno captante de $\left({ }^{131} \mathrm{I}\right)$ $\mathrm{NaI}$ foi descrito como causa de PCI após dose terapêutica de ( $\left.{ }^{131} \mathrm{I}\right) \mathrm{NaI}$ falso-positiva em 2003 por Gunawardana e cols., em caso de carcinoma folicular de tireoide (17). Esses autores não descrevem, no entanto, estudo citológico nem dosagem de $\mathrm{Tg}$ em conteúdo aspirado, tendo sido o diagnóstico de cisto hepático simples, firmado apenas com imagem de TC (17). Várias outras lesões hepáticas como causas de PCI falsos-positivas, como cisto hidático, dilatação de ducto intra-hepático e abscesso hepático, também já foram publicadas em forma de relato de caso $(16,18,19)$.

No caso descrito, diante da necessidade da quarta dose de ( ${ }^{131} \mathrm{I}$ ) $\mathrm{NaI}$ (dose acumulada de $900 \mathrm{mCi}, 33300$ $\mathrm{MBq}$ ), foi utilizado o lítio como adjuvante à terapia com ( $\left.{ }^{131} \mathrm{I}\right) \mathrm{NaI}$, na tentativa de ampliar a retenção do (131I) NaI nas supostas lesões metastáticas e/ou residuais de CDT que pudessem ainda existir, otimizando o tratamento. Estudos que defendem tal uso baseiamse no conhecimento de que o lítio possui a capacidade de atrasar a liberação do iodo, embora não modifique a quantidade captada pelas células (20).

Ressalta-se que, diante do aspecto benigno da lesão hepática em estudos de imagem com US, TC e RNM de abdômen, além da queda do nível de Tg, depois de cada tratamento com ( $\left.{ }^{131} \mathrm{I}\right) \mathrm{NaI}$, somados ao resultado citológico favorável e à ausência de $\mathrm{Tg}$ no material adquirido da punção cística guiada por US, fica claro o diagnóstico de cisto hepático simples benigno, e não de metástase hepática do CPT, apesar da persistente captação de ( $\left.{ }^{131} \mathrm{I}\right) \mathrm{NaI}$ nas PCI após dose terapêutica. Enfatiza-se que essa entidade necessita ser amplamente reconhecida, em razão das sérias consequências de ser erroneamente diagnosticada como metástase hepática de carcinoma diferenciado de tireoide.

Uma vez que a lesão hepática foi a única imagem captante na última PCI após dose terapêutica, considera-se o resultado dessa como "negativo", ou melhor, falsopositivo. A PCI após dose terapêutica de ( $\left.{ }^{131} \mathrm{I}\right) \mathrm{NaI}$ negativa associada à elevação da $\mathrm{Tg}$ poderia ser explicada por possível desdiferenciação das células tumorais que perderiam, assim, a capacidade de concentrar iodo (15). PCI iodonegativas com níveis crescentes de $\mathrm{Tg}$, seja com TSH suprimido, não suprimido ou com estimulação exógena com TSH recombinante, podem ser investigadas com o emprego de outros marcadores. O cloreto de tálio (Tl 201) e o sestamibi [Tc 99m] podem ser empregados na tentativa de demonstrar doença residual 
em atividade. No entanto, são raramente utilizados em razão de possuírem menor sensibilidade e especificidade. Outra possibilidade é a utilização de octreotide - In ${ }^{111}$ para demonstração de lesões que possuem alta densidade de receptores de somatotastina, com a vantagem de não ser necessária nenhuma preparação prévia para realização desse exame. Estudos têm demonstrado o valor da tomografia por emissão de pósitrons (PET Scan), que utiliza a glicose marcada com flúor-18 (fluorodeoxiglicose $18 \mathrm{~F}$ ), para localizar recidivas e/ou metástases de CDT em 87,5\% dos pacientes com níveis de Tg elevadas e PCI após dose terapêutica negativa (1-3,9-13,15). Esse método é particularmente útil para a detecção das células tumorais menos diferenciadas que apresentam elevada taxa de consumo de glicose e não captam iodo (15). Todavia, a baixa disponibilidade e, principalmente, o custo desse exame ainda limitam sua utilização. Apesar disso, a possibilidade de sua realização não foi descartada, caso persista elevação da $\mathrm{Tg}$, para avaliar-se a necessidade de nova dose de ( $\left.{ }^{131} \mathrm{I}\right) \mathrm{NaI}$, além dos $900 \mathrm{mCi}(33300$ $\mathrm{MBq})$ já administrados.

Concluiu-se que, apesar de eficazes na maioria dos casos, os métodos de seguimento disponíveis atualmente enfrentam limitações que, caso não sejam reconhecidas, podem comprometer o tratamento dos CDT. Logo, a análise dos resultados proporcionados por esses exames deve ser feita de forma crítica.

Agradecimentos: ao Bernardo Lopes Cançado Fonseca, pelo auxílio na descrição das imagens radiológicas, e ao Márcio Weissheimer Lauria, pela colaboração na busca bibliográfica.

Declaração: os autores declaram não haver conflitos de interesse científico neste estudo.

\section{REFERÊNCIAS}

1. Tuttle RM, Leboeuf R, Martorella AJ. Papillary thyroid cancer: monitoring and therapy. Endocrinol Metab Clin N Am. 2007;36(3):753-78.

2. Maia AL, Ward LS, Carvalho GS, Graf H, Maciel RMB, Maciel LMZ, et al. Nódulos de tireóide e câncer diferenciado de tireóide: Consenso Brasileiro. Arq Bras Endocrinol Metab. 2007;51(5):867-93.

3. Schlumbeuger MJ, Filetti S, Hay ID. Nontoxic diffuse and nodular goiter and thyroid neoplasic. $11^{\text {th }}$ ed. Philadelphia: Saunders; 2008. p. 411-42.
4. Haq M, Harmer C. Differentiated thyroid carcinoma with distant metastases at presentation: prognostic factors and outcome. Clin Endocrinol (Oxf). 2005;63(1):87-93.

5. Hod N, Hagag P, Sandbank J, HorneT. Epidemiological characteristics and I-131 scintigraphic of patients with papillary thyroid carcinoma with distant metastases. Harefuah. 2001;140(12):1134-8, 1231-2.

6. Carling T, Ocal IT, Udelsman R. Special variants of differentiated thyroid cancer: does it alter the extent of surgery versus welldifferentiated thyroid cancer? World J Surg. 2007;31(5):916-23.

7. McElvanna K, McCusker G, Stirling I. Diffuse sclerosing variant of papillary thyroid carcinoma - a rare cause of goitre in a young patient. Ulster Med J. 2007;76(2):113- 4.

8. Falvo L, Giacomelli L, D'Andrea V, Marzulho A, Guerriero G, de Antoni E. Prognostic importance of sclerosing variant in papillary thyroid carcinoma. Am Surg. 2006;72(5):438-44.

9. Camargo RYA, Tomimori EK. Uso da ultra-sonografia no diagnóstico e seguimento do carcinoma bem diferenciado da tireóide. Arq Bras Endocrinol Metab. 2007;51(5):783-92.

10. Brucker-Davis F, Reynalds JC, Skarulis MC, Fraker DC, Alexander HR, Weintraub BD, et al. False-positive iodine-131 wholebody scans due to cholecystitis and sebaceous cyst. J Nucl Med. 1996;37(10):1690-93.

11. Mitchell G, Pratt BE, Vini L, McCready VR, Harmer C. False positive ${ }^{131} \mathrm{l}$ whole body scans in thyroid cancer. British J Radiol. 2000;73:627-35.

12. Leitha T, Staudenherz A. Frequency of dilemmas in ${ }^{131} \mid$ whole body scanning. Nuklearmedizin. 2003;42(2):55-62.

13. Cooper DS, Doherty GM, Haugen BR, Kloos RT, Lee SL, Mandel SJ, Mazzaferri EL, Mclver B, Sherman SI,Tuttle RM; The American Thyroid Association Guidelines Taskforce. Management guidelines for patients with thyroid nodules and differentiated thyroid cancer. Thyroid. 2006;16(2):1-23.

14. MacedoTAA, Chammas MC. Fígado. $2^{\text {th }}$ ed. Revinter. 2009. p. 69-70.

15. Yamaga LYI, Cunha ML, Wagner J, Thom AF, Daniel MM, Funari MBG. Valor diagnóstico da tomografia por emissão de pósitrons/ tomografia computadorizada (PET-CT) com 18fluor-deoxiglicose $\left(F^{-18} \mathrm{~F}\right)$ em pacientes com carcinoma diferenciado da tireóide, níveis séricos de tireoglobulina elevados e pesquisa de corpo inteiro com iodo negativa. Arq Bras Endocrinol Metab. 2007;51(4):581-6.

16. You DL, Tzen KY, Chen JF, Tsai MF. False-positive whole-body iodine-131 scan due to intrahepatic duct dilatation. J Nucl Med. 1997;38(12):1977-9.

17. Gunawardana DH, Pitman AG, Lichtenstein M. Benign hepatic cyst mimicking a funcional thyroid carcinoma metastasis on whole-body ${ }^{131}$ imaging. Clin Nucl Med. 2003;28(6):527-8.

18. Omür O, Ozbek SS, Akgün A, Yazici B, Mutlukoca N, Ozcan Z. False-positive I-131 acumulation in hepatic hydatid cyst. Clin Nucl Med. 2007;32(12):930-2.

19. Pena Pardo FJ, Crespo de la Jara A, Fernández Morejón FJ, Sureda González M, Forteza Vila J, Brugarolas Masllorens A. Solitary focus in the liver in a thyroid cancer patient after a whole body scan with 131 iodine. Rev Esp Med Nucl. 2007;26(5):294-6.

20. Koong SS, Reynolds JC, Movius EG, Keenan AM, Ain KB, Lakshmanan MC, et al. Lithium as a potential adjuvant to ${ }^{131}$ l therapy of metastatic, well differentiated thyroid carcinoma. J Clin Endocrinol Metab. 1999;84(3):912-6. 